\title{
Bifurcation and Chaotic Behavior of a Discrete-Time SIS Model
}

\author{
Junhong Li and Ning Cui \\ Department of Mathematics and Sciences, Hebei Institute of Architecture and Civil Engineering, Zhangjiakou, Hebei 075000, China
}

Correspondence should be addressed to Junhong Li; lijunhong27@yahoo.com.cn

Received 5 January 2013; Accepted 8 April 2013

Academic Editor: Qingdu Li

Copyright (C) $2013 \mathrm{~J}$. Li and N. Cui. This is an open access article distributed under the Creative Commons Attribution License, which permits unrestricted use, distribution, and reproduction in any medium, provided the original work is properly cited.

The discrete-time epidemic model is investigated, which is obtained using the Euler method. It is verified that there exist some dynamical behaviors in this model, such as transcritical bifurcation, flip bifurcation, Hopf bifurcation, and chaos. The numerical simulations, including bifurcation diagrams and computation of Lyapunov exponents, not only show the consistence with the theoretical analysis but also exhibit the rich and complex dynamical behaviors.

\section{Introduction}

Epidemic models have been widely used in different forms for studying epidemiological processes such as the spread of HIV [1], SARS [2], and influenza [3]. It is well known that dynamical systems with simple dynamical behavior in the constant parameter case display very complex behaviors including chaos when they are periodically perturbed $[4$, 5]. The continuous-time epidemic models have been widely investigated in many articles (e.g., [6-10] and the references cited therein). In recent years, we have found that more attention is paid to the discrete-time epidemic models (see [11-15] and the references cited therein). The reasons are as follows: first, difference models are more realistic than continuous differential ones because the epidemic statistics are compiled from given time intervals and are discontinuous. Second, the discrete-time models can provide natural simulators for the continuous cases. One can thus not only study the behaviors of the continuous-time model with good accuracy, but also assess the effect of lager time steps. At last, the use of discrete -time models makes it possible to use the entire arsenal of methods recently developed for the study of mappings and lattice equations, either from the integrability and/or chaos points of view.

On the other hand, daily treatments are frequently done for some infections, such as the group of those being responsible for the common cold, which do not confer any long lasting immunity. Such infections do not have a recovered state and individuals become susceptible again after infection.
For such reasons, according to [16], we firstly consider the SIS epidemic model with nonlinear incidence rate:

$$
\begin{gathered}
\frac{d S}{d t}=b S\left(1-\frac{S}{K}\right)-\beta S^{2} I+\gamma I, \\
\frac{d I}{d t}=\beta S^{2} I-d I-\gamma I,
\end{gathered}
$$

where $S$ denotes the susceptible population, $I$ is the infected population, and $b$ is the intrinsic birth rate constant. $K, \beta$ are the carrying capacity and the infection rate, respectively. $\gamma \geq$ 0 is the recovery rate constant $(1 / \gamma$ is the average infective time).

$$
S=\sqrt{\frac{d+\gamma}{\beta}} x, \quad I=\sqrt{\frac{d+\gamma}{\beta}} y, \quad d \tau=(d+\gamma) d t .
$$

We obtain the following system analogous to (1):

$$
\begin{gathered}
\frac{d x}{d \tau}=a_{0} x-a_{1} x^{2}-x^{2} y+a_{2} y, \\
\frac{d y}{d \tau}=x^{2} y-y,
\end{gathered}
$$

where

$$
a_{0}=\frac{b}{d+\gamma}, \quad a_{1}=\frac{b}{K \sqrt{\beta(d+\gamma)}}, \quad a_{2}=\frac{\gamma}{d+\gamma} .
$$


Applying Euler scheme to (3), we obtain the following equation:

$$
\begin{gathered}
x_{n+1}=\left(a_{0}+1\right) x_{n}-a_{1} x_{n}^{2}-x_{n}^{2} y_{n}+a_{2} y_{n}, \\
y_{n+1}=x_{n}^{2} y_{n} .
\end{gathered}
$$

This paper is organized as follows. In Section 2, we give sufficient conditions of existence for transcritical bifurcation, flip bifurcation, and Hopf bifurcation. In Section 3, a series of numerical simulations show that there are bifurcation and chaos in the discrete-time epidemic model. Finally, we give remarks to conclude this paper in Section 4.

\section{Bifurcations}

It is easy to visualize that system (5) has three fixed points $P_{0}(0,0), P_{1}\left(a_{0} / a_{1}, 0\right)$, and $P_{2}\left(1,\left(a_{0}-a_{1}\right) /\left(1-a_{2}\right)\right)$ when $a_{1}, a_{0}$ are fixed. We can see that the fixed point $P_{0}(0,0)$ is a saddle. In the following paper, we focus on investigating the bifurcations of $P_{1}, P_{2}$.

Theorem 1. If $a_{0}=a_{1}$ and $a_{0} \neq 2$, (5) undergo a transcritical bifurcation at $P_{1}$. Furthermore, the system has three fixed points when $a_{0}>a_{1}$ and has two fixed points when $a_{0} \leq a_{1}$.

Proof. The Jacobian matrix of (5) at $P_{1}$ takes the form

$$
J\left(P_{1}\right)=\left(\begin{array}{cc}
1-a_{0} & a_{2}-\frac{a_{0}^{2}}{a_{1}^{2}} \\
0 & \frac{a_{0}^{2}}{a_{1}^{2}}
\end{array}\right) \text {. }
$$

$J\left(P_{1}\right)$ has eigenvalues $\lambda_{1}=1-a_{0}, \lambda_{2}=a_{0}^{2} / a_{1}^{2}$. And $a_{0} \neq 2$ implies that $\left|\lambda_{1}\right| \neq 1$.

Let

$$
\xi_{n}=x_{n}-\frac{a_{0}}{a_{1}}, \quad \eta_{n}=y_{n}, \quad c_{n}=a_{0}-a_{1},
$$

equation (5) becomes

$$
\begin{gathered}
\xi_{n+1}=\left(1-a_{1}\right) \xi_{n}+\left(a_{2}-1\right) \eta_{n}+F_{1}\left(\xi_{n}, \eta_{n}, c_{n}\right), \\
\eta_{n+1}=\eta_{n}+F_{2}\left(\xi_{n}, \eta_{n}, c_{n}\right), \\
c_{n+1}=c_{n},
\end{gathered}
$$

where

$$
\begin{gathered}
F_{1}\left(\xi_{n}, \eta_{n}, c_{n}\right)=-\left(a_{1}+\eta_{n}\right) \xi_{n}^{2}-\frac{2 \xi_{n} \eta_{n} c_{n}}{a_{1}} \\
-\left(2 \eta_{n}+c_{n}\right) \xi_{n}-\frac{\eta_{n} c_{n}^{2}}{a_{1}^{2}}-\frac{2 c_{n} \eta_{n}}{a_{1}}, \\
F_{2}\left(\xi_{n}, \eta_{n}, c_{n}\right)=2 \xi_{n} \eta_{n}+\frac{2 c_{n} \eta_{n}}{a_{1}}+\xi_{n}^{2} \eta_{n}+\frac{\eta_{n} c_{n}^{2}}{a_{1}^{2}}+\frac{2 \xi_{n} \eta_{n} c_{n}}{a_{1}} .
\end{gathered}
$$

By the following transformation:

$$
\left(\begin{array}{l}
\xi_{n} \\
\eta_{n} \\
c_{n}
\end{array}\right)=\left(\begin{array}{ccc}
1 & 1 & 0 \\
1 & \frac{a_{1}}{a_{2}-1} & 0 \\
0 & 0 & 1
\end{array}\right)\left(\begin{array}{l}
p_{n} \\
q_{n} \\
\sigma_{n}
\end{array}\right)
$$

equation (8) becomes

$$
\left(\begin{array}{c}
p_{n+1} \\
q_{n+1} \\
\sigma_{n+1}
\end{array}\right)=\left(\begin{array}{ccc}
1-a_{1} & 0 & 0 \\
0 & 1 & 0 \\
0 & 0 & 1
\end{array}\right)\left(\begin{array}{c}
p_{n} \\
q_{n} \\
\sigma_{n}
\end{array}\right)+\left(\begin{array}{c}
f_{1}\left(p_{n}, q_{n}, \sigma_{n}\right) \\
f_{2}\left(p_{n}, q_{n}, \sigma_{n}\right) \\
0
\end{array}\right)
$$

where

$$
\begin{aligned}
& f_{1}\left(p_{n}, q_{n}, \sigma_{n}\right) \\
& =\left(\frac{a_{1}+a_{2}-1}{1-a_{2}} q_{n}-a_{1}\right) p_{n}^{2} \\
& +\left[\frac{2\left(a_{1}+a_{2}-1\right)\left(\sigma_{n}+1\right)\left(q_{n}+q_{n}^{2}\right)}{a_{1}\left(1-a_{2}\right)}-\sigma_{n}-2 a_{1} q_{n}\right] p_{n} \\
& +\frac{2\left(a_{1}+a_{2}-1\right) q_{n}^{3}}{a_{1}\left(1-a_{2}\right)}+\left[\frac{2\left(a_{1}+a_{2}-1\right)\left(\sigma_{n}+a_{1}\right)}{a_{1}\left(1-a_{2}\right)}-a_{1}\right] q_{n}^{2} \\
& +\left[\frac{\left(a_{1} a_{2}+2 a_{2}-2\right) \sigma_{n}}{a_{1}\left(1-a_{2}\right)}+\frac{2\left(a_{1}+a_{2}-1\right) \sigma_{n}^{2}}{a^{3}\left(1-a_{2}\right)}\right] q_{n}, \\
& \quad+2 p_{n} q_{n}+\frac{2 q_{n} \sigma_{n}}{a_{1}}+\frac{\left(a_{1} p_{n}+\sigma_{n}\right)^{2} q_{n}}{a_{1}^{2}} .
\end{aligned}
$$

Then, we can consider

$$
\begin{aligned}
p_{n}=g\left(q_{n}, \sigma_{n}\right)= & \varepsilon_{1} q_{n}^{2}+\varepsilon_{2} q_{n} \sigma_{n}+\varepsilon_{3} \sigma_{n}^{2} \\
& +o\left(\left(\left|p_{n}\right|+\left|q_{n}\right|\right)^{3}\right)
\end{aligned}
$$

which must satisfy

$$
\begin{aligned}
g\left(q_{n+1}, \sigma_{n+1}\right) & =g\left(q_{n}+f_{2}\left(g\left(q_{n}, \sigma_{n}\right), q_{n}, \sigma_{n}\right), \sigma_{n+1}\right) \\
& =\left(1-a_{1}\right) g\left(q_{n}, \sigma_{n}\right)+f_{1}\left(g\left(q_{n}, \sigma_{n}\right), q_{n}, \sigma_{n}\right) .
\end{aligned}
$$

Thus, we have

$$
\varepsilon_{1}=\frac{a_{1} a_{2}+2 a_{2}-2}{a_{1}\left(1-a_{2}\right)}, \quad \varepsilon_{2}=\frac{a_{1} a_{2}+2 a_{2}-2}{a_{1}^{2}\left(1-a_{2}\right)}, \quad \varepsilon_{3}=0 .
$$


And (8) is restricted to the center manifold, which is given by

$$
\begin{aligned}
f: q_{n+1}= & \frac{2\left(a_{1} a_{2}+2 a_{2}-2\right)}{a_{1}\left(1-a_{2}\right)}\left(q_{n}^{3}+\frac{q_{n}^{2} \sigma_{n}}{a_{1}}\right) \\
& +q_{n}^{3}+2 q_{n}^{2}+q_{n}+\frac{2 q_{n}^{2} \sigma_{n}}{a_{1}}+\frac{2 q_{n} \sigma_{n}}{a_{1}} \\
& +o\left(\left(\left|q_{n}\right|+\left|\sigma_{n}\right|\right)^{4}\right) .
\end{aligned}
$$

Since

$$
\begin{gathered}
f\left(0, \sigma_{n}\right)=0,\left.\quad \frac{\partial f}{\partial q}\right|_{(0,0)}=1,\left.\quad \frac{\partial^{2} f}{\partial q^{2}}\right|_{(0,0)}=4, \\
\left.\frac{\partial^{2} f}{\partial q \partial \sigma}\right|_{(0,0)}=-\frac{2}{a_{1}} \neq 0,
\end{gathered}
$$

system (5) undergoes a transcritical bifurcation at $P_{1}$. This proves the theorem.

Theorem 2. Equation (5) undergoes a flip bifurcation at $P_{1}$ when $a_{0}=2, a_{1} \neq a_{0}$. Furthermore, the stable periodic- 2 point bifurcates from this fixed point.

Proof. Let

$$
\xi_{n}=x_{n}-\frac{a_{0}}{a_{1}}, \quad \rho_{n}=y_{n}, \quad c_{n}=a_{0}-2,
$$

system (5) becomes

$$
\begin{gathered}
\xi_{n+1}=-\xi_{n}+\left(a_{2}-\frac{4}{a_{1}^{2}}\right) \rho_{n}+F_{1}\left(\xi_{n}, c_{n}, \rho_{n}\right), \\
c_{n+1}=-c_{n}, \\
\rho_{n+1}=\frac{4}{a_{1}^{2}} \rho_{n}+F_{2}\left(\xi_{n}, c_{n}, \rho_{n}\right),
\end{gathered}
$$

where

$$
\begin{aligned}
F_{1}\left(\xi_{n}, c_{n}, \rho_{n}\right)= & -\left(a_{1}+\rho_{n}\right) \xi_{n}^{2} \\
& -\left(c_{n}+\frac{2\left(c_{n}+2\right) \rho_{n}}{a_{1}}\right) \xi_{n}-\frac{4 c_{n}+c_{n}^{2}}{a_{1}^{2}} \rho_{n}, \\
F_{2}\left(\xi_{n}, c_{n}, \rho_{n}\right)= & \rho_{n} \xi_{n}^{2}+\frac{4 c_{n}+c_{n}^{2}}{a_{1}^{2}} \rho_{n}+\frac{2\left(c_{n}+2\right) \rho_{n}}{a_{1}} \xi_{n} .
\end{aligned}
$$

By the following transformation:

$$
\left(\begin{array}{l}
\xi_{n} \\
c_{n} \\
\rho_{n}
\end{array}\right)=\left(\begin{array}{ccc}
1 & 0 & 1 \\
0 & 1 & 0 \\
& & \frac{\left(a_{1}^{2}+4\right)}{\left(a_{2} a_{1}^{2}-4\right)}
\end{array}\right)\left(\begin{array}{l}
\phi_{n} \\
u_{n} \\
v_{n}
\end{array}\right),
$$

equation (19) becomes

$$
\left(\begin{array}{l}
\phi_{n+1} \\
u_{n+1} \\
v_{n+1}
\end{array}\right)=\left(\begin{array}{ccc}
-1 & 0 & 0 \\
0 & -1 & 0 \\
0 & 0 & \frac{4}{a_{1}^{2}}
\end{array}\right)\left(\begin{array}{l}
\phi_{n} \\
u_{n} \\
v_{n}
\end{array}\right)+\left(\begin{array}{c}
f_{1}\left(\phi_{n}, u_{n}, v_{n}\right) \\
0 \\
f_{2}\left(\phi_{n}, u_{n}, v_{n}\right)
\end{array}\right),
$$

where

$$
\begin{gathered}
f_{1}\left(\phi_{n}, u_{n}, v_{n}\right) \\
=\left(\frac{a_{1}^{2}+a_{2} a_{1}^{2}}{4-a_{2} a_{1}^{2}} v_{n}-a_{1}\right) \phi_{n}^{2} \\
+\left[\frac{2\left(1+a_{2}\right) a_{1}^{2} v_{n}^{2}}{4-a_{2} a_{1}^{2}}\right. \\
\left.+\frac{2 a_{1}\left(u_{n}-2+a_{2} a_{1}^{2}+a_{2} u_{n}+2 a_{2}\right) v_{n}}{4-a_{2} a_{1}^{2}}-u_{n}\right] \phi_{n} \\
+\frac{a_{1}^{2}+a_{2} a_{1}^{2}}{4-a_{2} a_{1}^{2}} v_{n}^{3}+\frac{a_{1}\left(a_{2} a_{1}^{2}+2 a_{2} u_{n}+4 a_{2}+2 u_{n}\right)}{4-a_{2} a_{1}^{2}} v_{n}^{2} \\
+\frac{\left(1+a_{2}\right) u_{n}^{2} v_{n}}{4-a_{2} a_{1}^{2}}+\frac{a_{2}\left(4+a_{1}^{2}\right) u_{n} v_{n}}{4-a_{2} a_{1}^{2}}, \\
f_{2}\left(\phi_{n}, u_{n}, v_{n}\right)=v_{n}^{3}+2\left(\phi_{n}+\frac{u_{n}+4}{a_{1}}\right) v_{n}^{2} \\
+\frac{\left(a_{1} \phi_{n}+u_{n}\right)\left(a_{1} \phi_{n}+u_{n}+4\right) v_{n}}{a_{1}^{2}} .
\end{gathered}
$$

Then, we can consider $v_{n}=g\left(\phi_{n}, u_{n}\right)=\varepsilon_{1} \phi_{n}^{2}+\varepsilon_{2} \phi_{n} u_{n}+\varepsilon_{3} u_{n}^{2}+$ $o\left(\left(\left|\phi_{n}\right|+\left|u_{n}\right|\right)^{3}\right)$, which must satisfy

$$
\begin{aligned}
g\left(-\phi_{n}\right. & \left.+f_{1}\left(\phi_{n}, g\left(\phi_{n}, u_{n}\right), v_{n}\right), u_{n+1}\right) \\
= & \frac{4}{a_{1}^{2}} g\left(\phi_{n}, u_{n}\right)+g^{3}\left(\phi_{n}, u_{n}\right) \\
& +2\left(\phi_{n}+\frac{u_{n}+4}{a_{1}}\right) g^{2}\left(\phi_{n}, u_{n}\right) \\
& +\frac{\left(a_{1} \phi_{n}+u_{n}\right)\left(a_{1} \phi_{n}+u_{n}+4\right) g\left(\phi_{n}, u_{n}\right)}{a_{1}^{2}} .
\end{aligned}
$$

Thus, we have $\varepsilon_{k}=0, k=1,2,3$. We obtain the center manifold as follows:

$$
\begin{aligned}
v_{n}= & g\left(\phi_{n}, u_{n}\right)=\varepsilon_{4} \phi_{n}^{2} u_{n}+\varepsilon_{5} \phi_{n} u_{n}^{2} \\
& +\varepsilon_{6} u_{n}^{3}+o\left(\left(\left|\phi_{n}\right|+\left|u_{n}\right|\right)^{4}\right) .
\end{aligned}
$$

And (19) is restricted to the center manifold, which is given by

$$
f: \phi_{n+1}=-\phi_{n}+f_{1}\left(\phi_{n}, g\left(\phi_{n}, u_{n}\right), v_{n}\right) .
$$


Direct calculations show that

$$
\begin{gathered}
\left.\left(\frac{\partial f}{\partial \sigma} \frac{\partial^{2} f}{\partial p^{2}}+2 \frac{\partial^{2} f}{\partial p \partial \sigma}\right)\right|_{(0,0)}=-2 \\
\left.\left(\frac{1}{2}\left(\frac{\partial^{2} f}{\partial p^{2}}\right)^{2}+\frac{1}{3}\left(\frac{\partial^{3} f}{\partial p^{3}}\right)\right)\right|_{(0,0)}=\frac{a_{1}^{2}}{2}
\end{gathered}
$$

Hence, system (5) undergoes a flip bifurcation at $P_{1}$. This completes the proof.

The positive fixed point is so important to the biological system that people usually are very interested in it. We will next pay attention to the only positive fixed point $P_{2}$ of (5).

Theorem 3. Equation (5) undergoes a transcritical bifurcation at $P_{2}$ when $a_{1}=a_{0}, a_{1} \neq 2$, and $\Delta>0$, where

$$
\Delta=\frac{\left(1+a_{2}\right)^{2} a_{0}^{2}}{\left(1-a_{2}\right)^{2}}+8\left(a_{1}-a_{0}\right)+\frac{4 a_{1}^{2} a_{2}^{2}-4 a_{2}\left(a_{2}+1\right)}{\left(1-a_{2}\right)^{2}} .
$$

Theorem 4. If $\Delta>0, a_{1} \neq a_{0}$, and $a_{0}=\left(2-2 a_{2}-a_{1}+\right.$ $\left.3 a_{1} a_{2}\right) / 2 a_{2}$, (5) undergoes a flip bifurcation at $P_{2}$.

Since the analysis is similar to the case at $P_{1}$, the previously mentioned proofs are omitted.

We next give the condition of existence of Hopf bifurcation by using the hopf bifurcation theorem in [17].

The characteristic equation of the Jacobian matrix $J\left(P_{2}\right)$ can be written as $\lambda^{2}-t_{2} \lambda+d_{2}=0$, where

$$
\begin{gathered}
t_{2}=2+a_{0}-2 a_{1}-\frac{2\left(a_{0}-a_{1}\right)}{1-a_{2}}, \\
d_{2}=t_{2}-1+2 a_{0}-2 a_{1} .
\end{gathered}
$$

The eigenvalues of the Jacobian matrix of (5) at $P_{2}$ are $\lambda_{1,2}=\left(t_{2} \pm \sqrt{t_{2}^{2}-4 d_{2}}\right) / 2$. The eigenvalues $\lambda_{1,2}$ are complex conjugates for $\Delta<0$. We translate the fixed point $P_{2}\left(1,\left(a_{0}-\right.\right.$ $\left.\left.a_{1}\right) /\left(1-a_{2}\right)\right)$ to the origin by $x_{n}=\varphi_{n}-1, y_{n}=\delta_{n}-\left(\left(a_{0}-\right.\right.$ $\left.a_{1}\right) /\left(1-a_{2}\right)$ ), and the system (5) becomes

$$
\begin{gathered}
\varphi_{n+1}=\left(t_{2}-1\right) \varphi_{n}+\left(a_{2}-1\right) \delta_{n} \\
-\left(\delta_{n}+\frac{a_{0}-a_{1} a_{2}}{1-a_{2}}\right) \varphi_{n}^{2}-2 \varphi_{n} \delta_{n}, \\
\delta_{n+1}=\frac{2\left(a_{0}-a_{1}\right)}{1-a_{2}} \varphi_{n}+\delta_{n}+2 \varphi_{n} \delta_{n}+\left(\delta_{n}+\frac{a_{0}-a_{1}}{1-a_{2}}\right) \varphi_{n}^{2} .
\end{gathered}
$$

The eigenvalues of the matrix associated with the linearized map $(30)$ at fixed point $(0,0)$ are complex conjugates which are written as $\lambda, \bar{\lambda}=\left(t_{2} \pm i \sqrt{4 d_{2}-t_{2}^{2}}\right) / 2$, where

$$
|\lambda|=\sqrt{1+3 a_{0}-4 a_{1}-\frac{2\left(a_{0}-a_{1}\right)}{1-a_{2}}} .
$$

Now assume that $3 a_{2}<1$, and let $a_{00}=\left(2 a_{1}\left(1-2 a_{2}\right)\right) /(1-$ $\left.3 a_{2}\right)$. Then we have

$$
\begin{gathered}
\left.\frac{d(|\lambda|)}{d a_{0}}\right|_{a_{0}=a_{00}}=\frac{1-3 a_{2}}{2\left(1-a_{2}\right)}>0, \quad\left|\lambda\left(a_{10}\right)\right|=1, \\
\lambda\left(a_{00}\right)=1+\frac{a_{1} a_{2}-a_{1}}{1-3 a_{2}} \\
\pm i \frac{\sqrt{a_{1}\left(1-a_{2}\right)\left(2-a_{1}+a_{1} a_{2}-6 a_{2}\right)}}{1-3 a_{2}}, \\
\lambda^{j} \neq 1, \quad j=1,2,3,4 .
\end{gathered}
$$

Let

$$
\begin{gathered}
\mathrm{T}=\left(\begin{array}{cc}
a_{2}-1 & 0 \\
\frac{2-t_{2}}{2} & -\frac{\sqrt{4 d_{2}-t_{2}^{2}}}{2}
\end{array}\right), \\
\left(\begin{array}{l}
\varphi_{n} \\
\delta_{n}
\end{array}\right)=\mathrm{T}\left(\begin{array}{l}
\phi_{n} \\
\sigma_{n}
\end{array}\right) .
\end{gathered}
$$

The system (30) becomes

$$
\begin{aligned}
\left(\begin{array}{l}
\phi_{n+1} \\
\sigma_{n+1}
\end{array}\right)= & \left(\begin{array}{cc}
\frac{t_{2}}{2} & -\frac{\sqrt{4 d_{2}-t_{2}^{2}}}{2} \\
\frac{\sqrt{4 d_{2}-t_{2}^{2}}}{2} & \frac{t_{2}}{2}
\end{array}\right)\left(\begin{array}{l}
\phi_{n} \\
\sigma_{n}
\end{array}\right) \\
& +\left(\begin{array}{l}
f_{1}\left(\phi_{n}, \sigma_{n}\right) \\
f_{2}\left(\phi_{n}, \sigma_{n}\right)
\end{array}\right),
\end{aligned}
$$

where

$$
\begin{aligned}
f_{1}\left(\phi_{n}, \sigma_{n}\right) & \\
= & \left(\frac{a_{0}+a_{0} a_{2}}{2}-a_{1} a_{2}\right) \phi_{n}^{3} \\
& +\left[\frac{\left(a_{2}-1\right) \sqrt{4 d_{2}-t_{2}^{2}}}{2} \sigma_{n}+\frac{a_{2}\left(2 a_{0}-a_{1}-a_{1} a_{2}\right)}{a_{2}-1}\right] \phi_{n}^{2} \\
& +\sqrt{4 d_{2}-t_{2}^{2}} \sigma_{n} \phi_{n}, \\
f_{2}\left(\phi_{n}, \sigma_{n}\right) & \left(2-t_{2}\right)\left(2 a_{2}-t_{2}\right)\left(a_{2}-1\right) \\
= & 2 \sqrt{4 d_{2}-t_{2}^{2}} \\
& +\left(a_{n}^{3}-1\right)\left(a_{2}-\frac{t_{2}}{2}\right) \phi_{n}^{2} \sigma_{n}-\frac{\left(2-t_{2}\right)\left(2-t_{2}+a_{1} a_{2}-a_{0}\right)}{\sqrt{4 d_{2}-t_{2}^{2}}} \phi_{n}^{2} \\
& -\frac{2\left(a_{2}-1\right)\left(2-t_{2}-a_{0}+a_{1}\right)}{\sqrt{4 d_{2}-t_{2}^{2}} \phi_{n}^{2}+\left(2 a_{2}-t_{2}\right) \sigma_{n} \phi_{n} .} \\
& \\
& \\
& \\
&
\end{aligned}
$$


Notice that (34) is exactly on the center manifold in the form, in which the coefficient $l[18]$ is given by $l=-\operatorname{Re}[((1-$ $\left.\left.2 \lambda) \bar{\lambda}^{2} /(1-\lambda)\right) l_{11} l_{20}\right]-(1 / 2)\left|l_{11}\right|^{2}-\left|l_{02}\right|^{2}+\operatorname{Re}\left(\bar{\lambda} l_{21}\right)$, where

$$
\begin{aligned}
& l_{11}=\frac{1}{4}\left[\left(f_{1 \phi \phi}+f_{1 \sigma \sigma}\right)+\left(f_{2 \phi \phi}+f_{2 \sigma \sigma}\right) i\right] \\
& =\frac{3 a_{0} a_{2}-2 a_{1} a_{2}^{2}-a_{0}}{4 a_{2}-4}-\frac{1}{4 \sqrt{4 d_{2}-t_{2}^{2}}} \\
& \times\left[\left(4-2 t_{2}\right)\left(2-t_{2}+a_{1} a_{2}-a_{0}\right)\right. \\
& \left.+\left(a_{2}-1\right)\left(4-2 t_{2}-2 a_{0}+2 a_{1}\right)\right] i \\
& l_{20}=\frac{1}{8}\left[\left(f_{1 \phi \phi}-f_{1 \sigma \sigma}+2 f_{2 \phi \sigma}\right)+\left(f_{2 \phi \phi}-f_{2 \sigma \sigma}-2 f_{1 \phi \sigma}\right) i\right] \\
& =\frac{1}{4}\left(a_{2}-a_{1} a_{2}-2+2 a_{0}\right)-\frac{1}{8 \sqrt{4 d_{2}-t_{2}^{2}}} \\
& \times\left[\left(4-2 t_{2}\right)\left(2-t_{2}+a_{1} a_{2}-a_{0}\right)+\left(a_{2}-1\right)\right. \\
& \left.\times\left(4-2 t_{2}-2 a_{0}+2 a_{1}\right)-8 d_{2}+2 t_{2}^{2}\right] i, \\
& l_{02}=\frac{1}{8}\left[\left(f_{1 \phi \phi}-f_{1 \sigma \sigma}-2 f_{2 \phi \sigma}\right)+\left(f_{2 \phi \phi}-f_{2 \sigma \sigma}+2 f_{1 \phi \sigma}\right) i\right] \\
& =\frac{1}{8}\left(2 a_{0}-2 a_{0} a_{2}-2-4 a_{2}+3 t_{2}\right) \\
& +\frac{1}{8}\left(2 a_{0}-a_{1} a_{2}-2+t_{2}+2 \sqrt{4 d_{2}-t_{2}^{2}}\right) i, \\
& l_{21}=\frac{1}{16}\left[\left(f_{1 \phi \phi \phi}+f_{1 \phi \sigma \sigma}+f_{2 \phi \phi \sigma}+f_{2 \sigma \sigma \sigma}\right)\right. \\
& \left.+\left(f_{2 \phi \phi \phi}+f_{2 \phi \sigma \sigma}-f_{1 \phi \phi \sigma}-f_{1 \sigma \sigma \sigma}\right) i\right] \\
& =\frac{1}{8}\left(1-a_{2}\right)\left(3+a_{2}-2 t_{2}\right) \\
& +\frac{1}{8 \sqrt{4 d_{2}-t_{2}^{2}}}\left(6 a_{2}-3 t_{2}+3 a_{2} t_{2}+8 d_{2}+t_{2}^{2}\right) i .
\end{aligned}
$$

From the previous analysis, we have Theorem 5.

Theorem 5. System (5) undergoes a Hopf bifurcation at fixed point $P_{2}$, if $l \neq 0, \Delta<0$ and

$$
a_{1} \neq \frac{2\left(1-a_{2}\right)+2 a_{2} a_{1}}{1+a_{3}} .
$$

\section{Numerical Simulations}

With development of scientific computation, computer becomes a powerful tool to study nonlinear systems, especially for a system without explicit solution. It not only is able to explore new complex dynamical behaviors, for example, periodic orbits and chaos in different regions $[16$, 19 ], but also can do rigorous analysis by combining modern dynamical systems theory and reliable computation, for example, topological horseshoes [20-23]. In this section, we use the bifurcation diagrams, Lyapunov exponents, and phase portraits to illustrate the previous analytic results and find new dynamics of the model (5) as the parameters vary. The attractors of (5) are also given by using the method in [20]. The bifurcation parameters are considered in the following three cases:

(I) varying $a_{0}$ in range $1.6 \leq a_{0} \leq 2.2$ and fixing $a_{1}=0.9$, $a_{3}=0.01$

(II) varying $a_{1}$ in range $0.8 \leq a_{1} \leq 1.2$ and fixing $a_{0}=2$, $a_{3}=0.01$;

(III) varying $a_{2}$ in range $0.3 \leq a_{2} \leq 0.6$ and fixing $a_{0}=2$, $a_{1}=0.9$.

For case (I). The bifurcation diagram of (5) in $a_{0}-x_{n}$ and $a_{0}-y_{n}$ space for $a_{1}=0.9, a_{3}=0.01$ is given in Figures 1(a) and 1(b) to show the dynamical changes of susceptible and infective, respectively, as $a_{0}$ varies. There is obvious phenomenon of bifurcation when we select the stepsize to be $10^{-4}$. The spectrum of Lyapunov exponents of the system (5) with respect to parameter $a_{1}$ is given in Figure 1(c).

Moreover, we can see that the orbit with initial values $(1,1)$ approaches to the stable fixed point $P_{2}$ for $a_{0}<1.8$ approximately, and Hopf bifurcation occurs at $a_{0} \approx 1.8$. When increased to $a_{0} \approx 2.05$, (5) becomes stable. In Figures (b) and 1(c), we observe the period $-4,8$ windows within the chaotic regions and boundary crisis at $a_{0} \approx 2.05$. For $a_{0} \in$ $(1.95,2.05)$ the maximum Lyapunov exponents are positive which correspond to chaotic region. To well see the dynamics, the attractor in the system (5) and time series of $x_{n}$ and $y_{n}$ are given in Figures 1(d) and 1(e), respectively.

For case (II). Figure 2(a) is the bifurcation diagram of (5) for $x_{n}$ and $y_{n}$ and depicts that there are period $-4,5$ windows within the chaotic regions and boundary crisis at $a_{1} \approx$ 1. Figure 2(b) shows the spectrum of Lyapunov exponents of the system (5) with respect to parameter $a_{1}$. For $a_{1} \in$ $(0.85,0.95)$ the maximum Lyapunov exponents are positive which correspond to chaotic region.

For case (III). Figures 3(a) and 3(b) are the bifurcation diagrams of (5) for $x_{n}$ and $y_{n}$, respectively, and depict that there are period $-2,4$ windows within the chaotic regions and boundary crisis at $a_{2}=0.5$. Figure 3(c) shows the spectrum of Lyapunov exponents of (5) with respect to parameter $a_{2}$. Figure 3(d) is the attractor of (5) with $a_{2}=0.46$.

\section{Conclusion}

In this paper, we investigate the behaviors of a discrete-time SIS epidemic model with nonlinear incidence rate, and find many complex and new interesting dynamical phenomena. Without the recovery rate of infectious hosts, (1) becomes the SI model (see [16]). Our theoretical analysis and numerical simulations have demonstrated that the model exhibits the variety of dynamical behaviors, which includes that the discrete epidemic model undergoes transcritical bifurcation, 


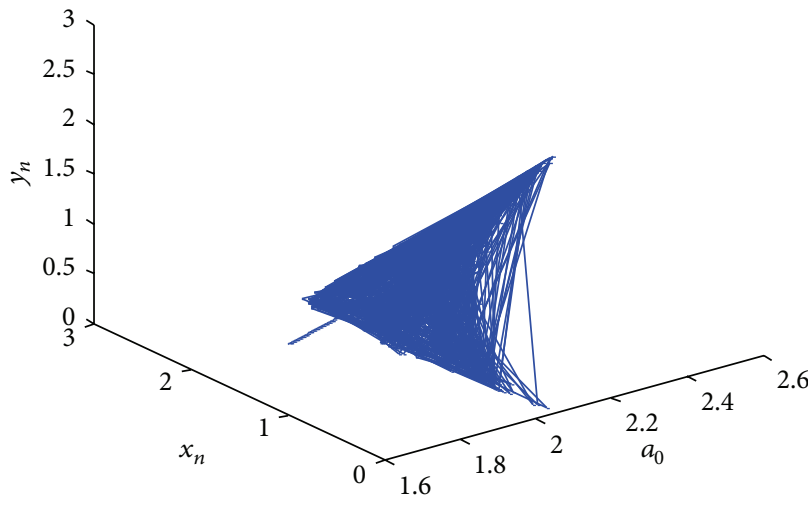

(a)

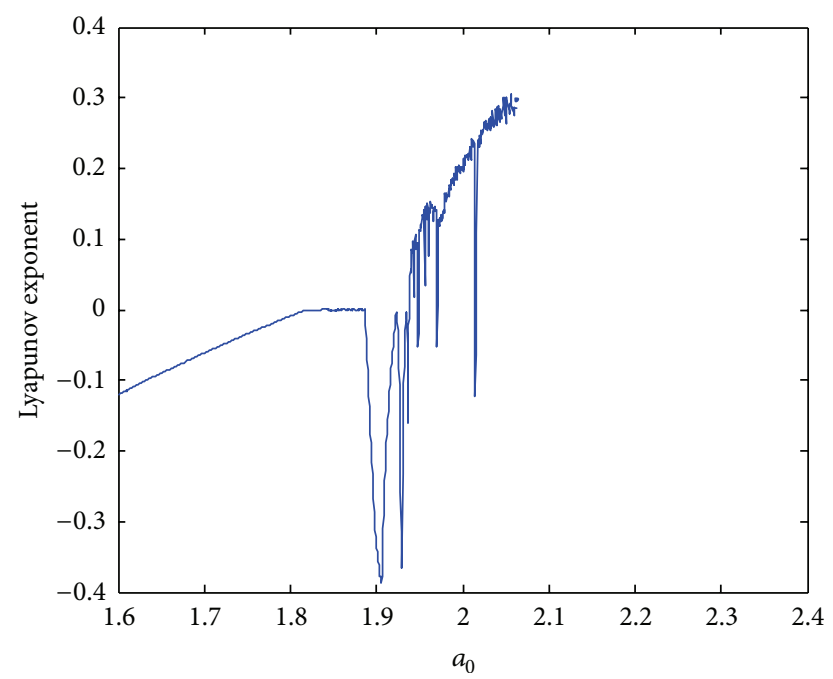

(c)
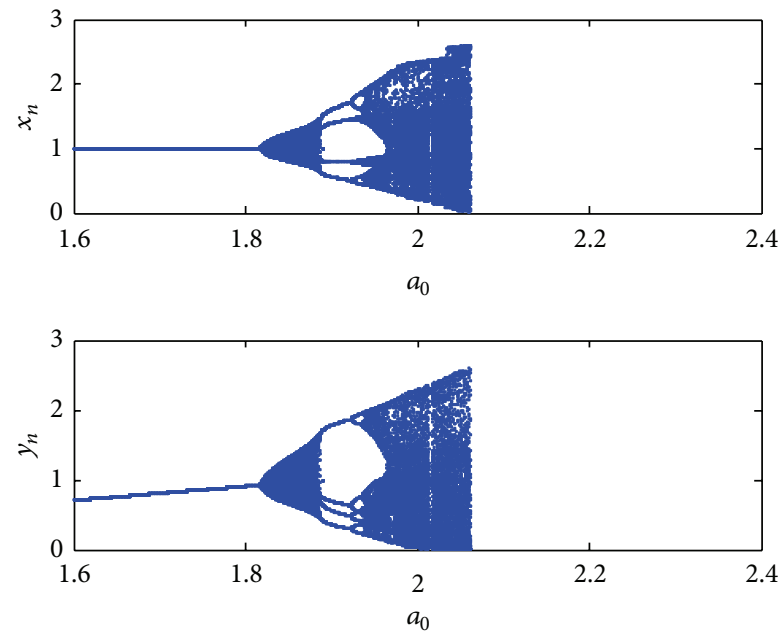

(b)

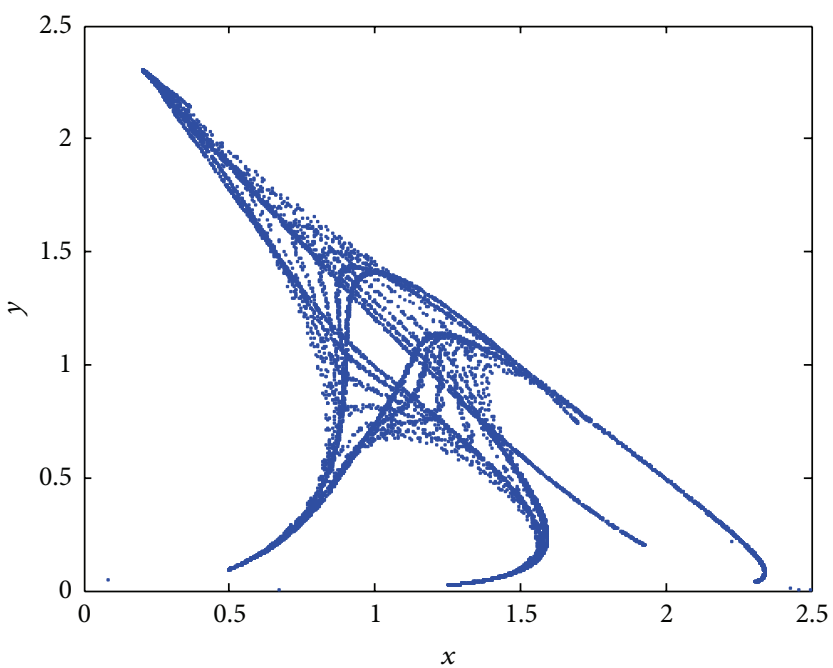

(d)
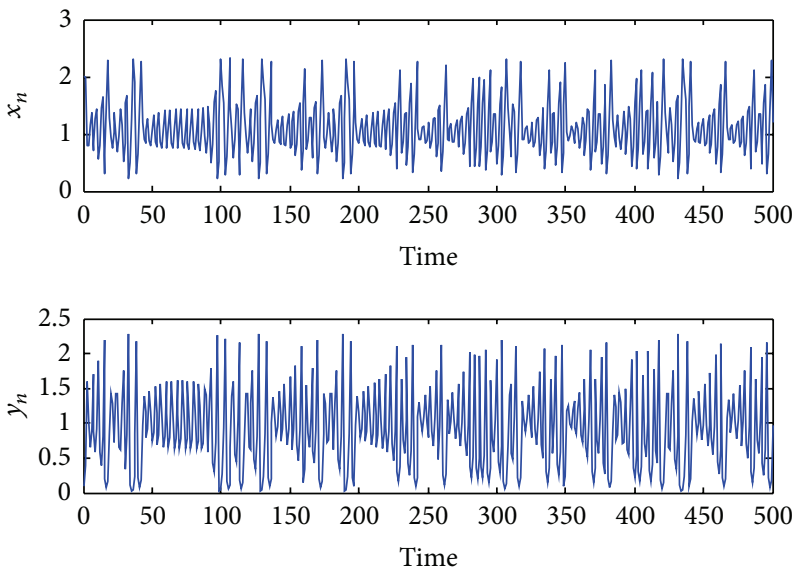

(e)

FIgURE 1: (a) Bifurcation diagram in $\left(a_{0}, x_{n}, y_{n}\right)$ space. (b) The bifurcation diagram in $a_{0}-x_{n}$ plane and in $a_{0}-y_{n}$ plane. (c) Spectrum of Lyapunov exponents corresponding to (b). (d) The attractor of (5) with $a_{0}=2$ (e) Time series of $x_{n}$ and $y_{n}$ of the same parameters. 

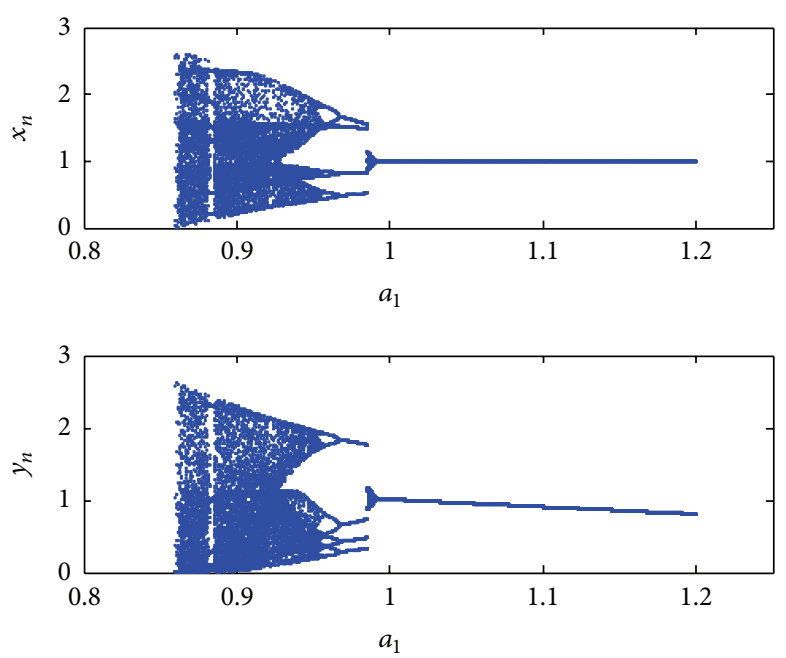

(a)

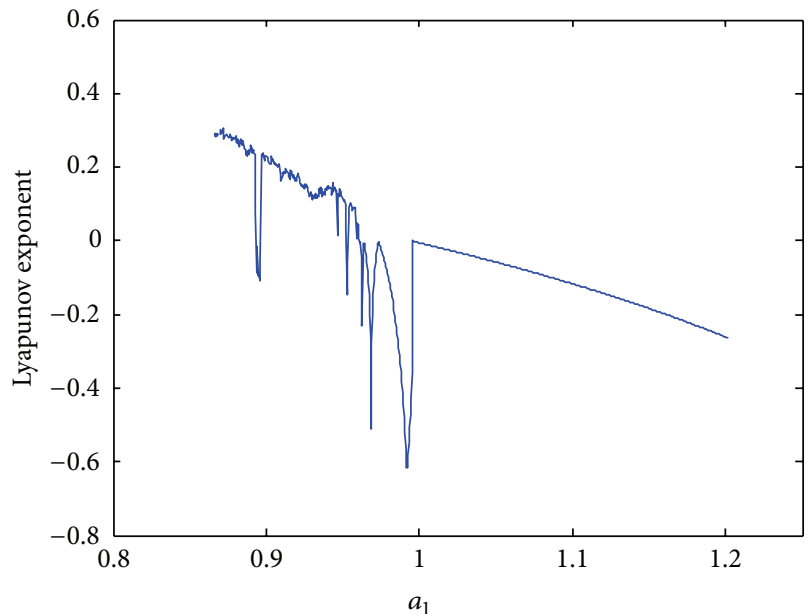

(b)

FIgURE 2

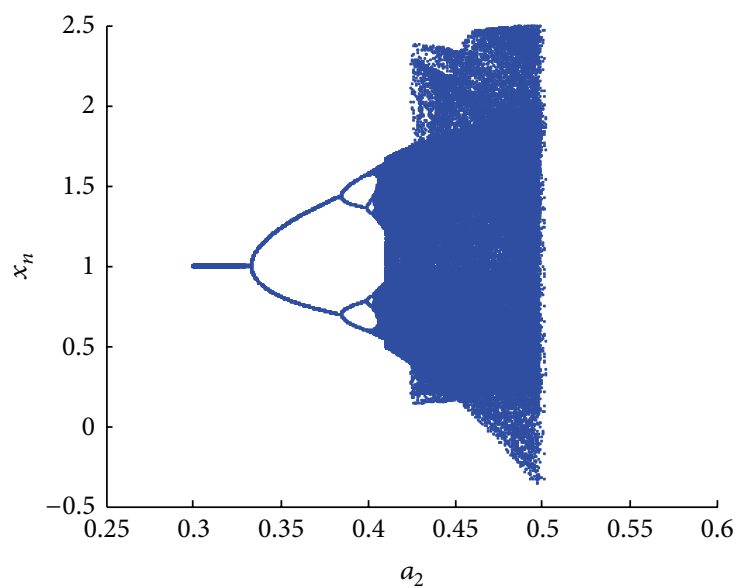

(a)

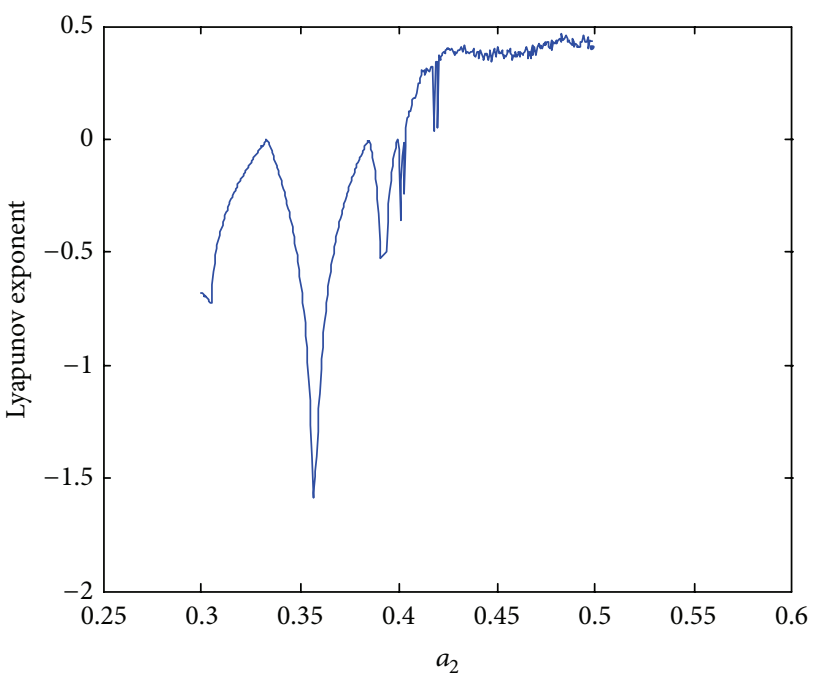

(c)

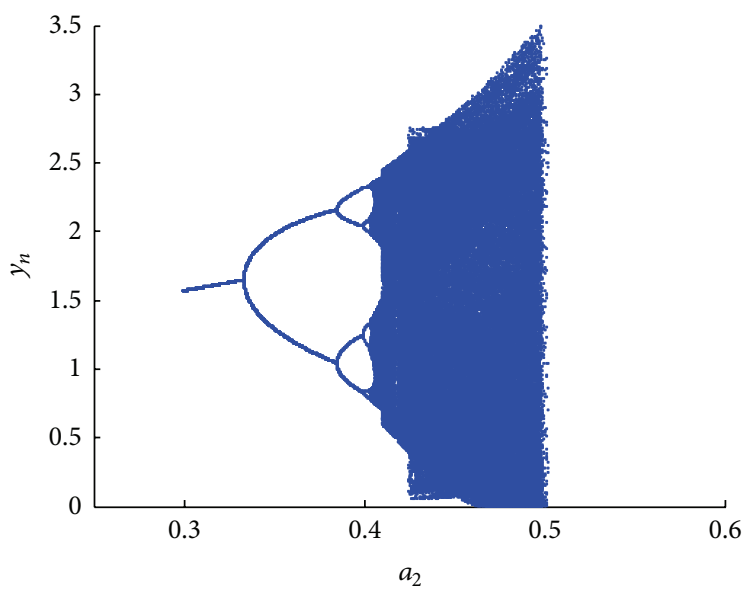

(b)

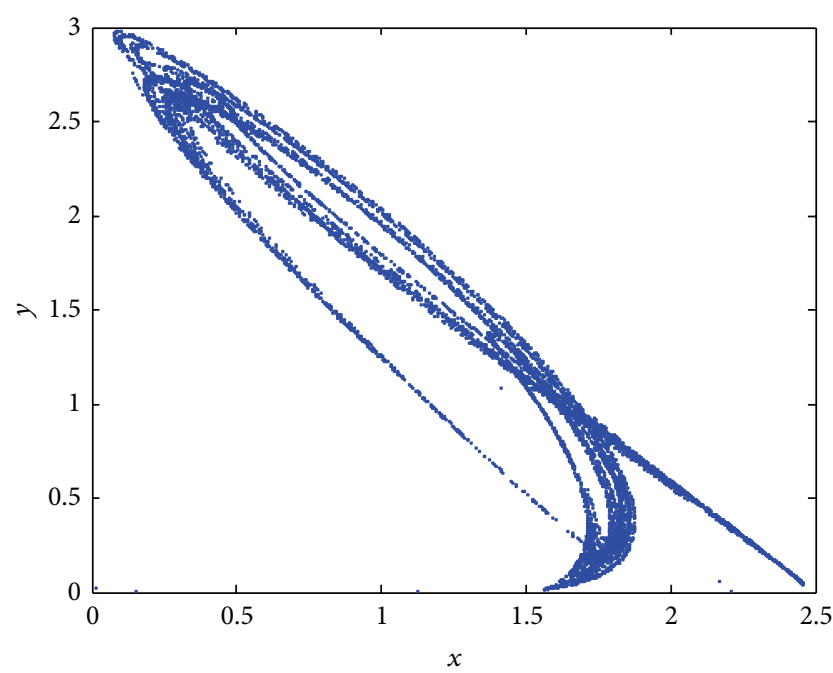

(d)

Figure 3 
flip bifurcation, Hopf bifurcation, and chaos. Furthermore, chaos can cause the population to run a higher risk of extinction due to the unpredictability $[24,25]$. Thus, how to control chaos in the epidemic model is very important, which needs further consideration.

\section{Acknowledgments}

The authors are very grateful to the reviewers for their valuable comments and suggestions. This work was supported by the Youth Science Foundations of Education Department of Hebei Province (nos. 2010233, 2011236).

\section{References}

[1] L. Cai, X. Li, M. Ghosh, and B. Guo, "Stability analysis of an HIV/AIDS epidemic model with treatment," Journal of Computational and Applied Mathematics, vol. 229, no. 1, pp. 313323, 2009.

[2] Y.-H. Hsieh, C.-C. King, C. W. S. Chen, M.-S. Ho, S.-B. Hsu, and Y.-C. Wu, "Impact of quarantine on the 2003 SARS outbreak: a retrospective modeling study," Journal of Theoretical Biology, vol. 244, no. 4, pp. 729-736, 2007.

[3] S. Iwami, Y. Takeuchi, X. Liu, and S. Nakaoka, "A geographical spread of vaccine-resistance in avian influenza epidemics," Journal of Theoretical Biology, vol. 259, no. 2, pp. 219-228, 2009.

[4] D. Summers, J. G. Cranford, and B. P. Healey, "Chaos in periodically forced discrete-time ecosystem models," Chaos, Solitons and Fractals, vol. 11, no. 14, pp. 2331-2342, 2000.

[5] J. Vandermeer, L. Stone, and B. Blasius, "Categories of chaos and fractal basin boundaries in forced predator-prey models," Chaos, Solitons and Fractals, vol. 12, no. 2, pp. 265-276, 2001.

[6] A. Gray, D. Greenhalgh, X. Mao, and J. Pan, "The SIS epidemic model with Markovian switching," Journal of Mathematical Analysis and Applications, vol. 394, no. 2, pp. 496-516, 2012.

[7] A. Gray, D. Greenhalgh, L. Hu, X. Mao, and J. Pan, "A stochastic differential equation SIS epidemic model," SIAM Journal on Applied Mathematics, vol. 71, no. 3, pp. 876-902, 2011.

[8] J. Liu and T. Zhang, "Bifurcation analysis of an SIS epidemic model with nonlinear birth rate," Chaos, Solitons \& Fractals, vol. 40, no. 3, pp. 1091-1099, 2009.

[9] D. Gao and S. Ruan, "An SIS patch model with variable transmission coefficients," Mathematical Biosciences, vol. 232, no. 2, pp. 110-115, 2011.

[10] Y. Li and J. Cui, "The effect of constant and pulse vaccination on SIS epidemic models incorporating media coverage," Communications in Nonlinear Science and Numerical Simulation, vol. 14, no. 5, pp. 2353-2365, 2009.

[11] Q. Wu and X. Fu, "Modelling of discrete-time SIS models with awareness interactions on degree-uncorrelated networks," Physica A, vol. 390, no. 3, pp. 463-470, 2011.

[12] P. L. Salceanu and H. L. Smith, "Persistence in a discrete-time stage-structured fungal disease model," Journal of Biological Dynamics, vol. 3, no. 2-3, pp. 271-285, 2009.

[13] J. E. Franke and A.-A. Yakubu, "Disease-induced mortality in density-dependent discrete-time S-I-S epidemic models," Journal of Mathematical Biology, vol. 57, no. 6, pp. 755-790, 2008.

[14] J. Li, Z. Ma, and F. Brauer, "Global analysis of discrete-time SI and SIS epidemic models," Mathematical Biosciences and Engineering, vol. 4, no. 4, pp. 699-710, 2007.
[15] L. J. S. Allen, Y. Lou, and A. L. Nevai, "Spatial patterns in a discrete-time SIS patch model," Journal of Mathematical Biology, vol. 58, no. 3, pp. 339-375, 2009.

[16] L. Li, G.-Q. Sun, and Z. Jin, "Bifurcation and chaos in an epidemic model with nonlinear incidence rates," Applied Mathematics and Computation, vol. 216, no. 4, pp. 1226-1234, 2010.

[17] J. Guckenheimer and P. Holmes, Nonlinear Oscillations, Dynamical Systems, and Bifurcations of Vector Fields, vol. 42, Springer, New York, NY, USA, 1983.

[18] S. Wiggins, Introduction to Applied Nonlinear Dynamical Systems and Chaos, vol. 2, Springer, New York, NY, USA, 1990.

[19] T. Chen, J. He, and Q. Yin, "Dynamics evolution of credit risk contagion in the CRT market," Dynamics in Nature and Society, vol. 2013, Article ID 206201, 9 pages, 2013.

[20] Q. Li, L. Zhang, and F. Yang, "An algorithm to automatically detect the Smale horseshoes," Discrete Dynamics in Nature and Society, Article ID 283179, 9 pages, 2012.

[21] Q. Li and X.-S. Yang, "A simple method for finding topological horseshoes," International Journal of Bifurcation and Chaos in Applied Sciences and Engineering, vol. 20, no. 2, pp. 467-478, 2010.

[22] X.-S. Yang, "Topological horseshoes and computer assisted verification of chaotic dynamics," International Journal of Bifurcation and Chaos in Applied Sciences and Engineering, vol. 19, no. 4, pp. 1127-1145, 2009.

[23] L. Qingdu and T. Song, "Algorithm for finding horseshoes in three-dimensinal hyperchaltic maps and its application," Acta Physica Sinica, vol. 62, no. 2, Article ID 020510, 2013.

[24] M. P. Hassell, H. N. Comins, and R. M. May, "Spatial structure and chaos in insect population dynamics," Nature, vol. 353, pp. 255-258, 1991.

[25] A. A. Berryman and J. A. Millstein, "Are ecological systems chaotic and if not, why not?" Trends in Ecology \& Evolution, vol. 4, no. 1, pp. 26-28, 1989. 


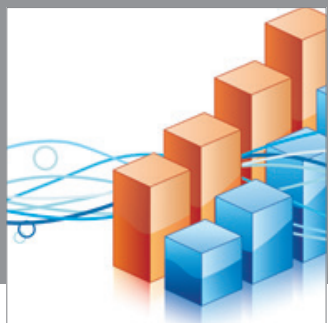

Advances in

Operations Research

mansans

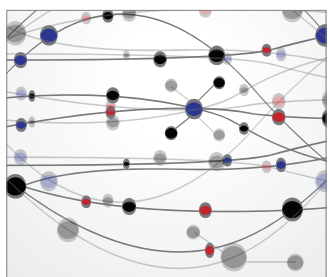

The Scientific World Journal
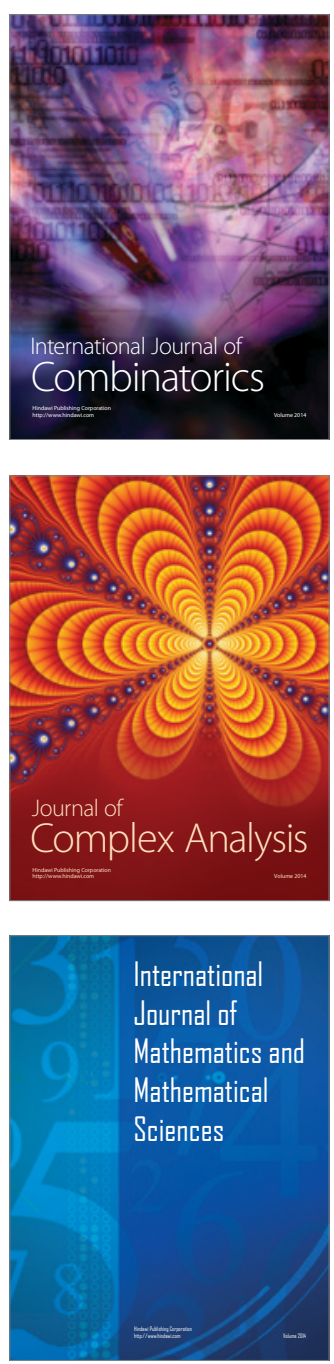
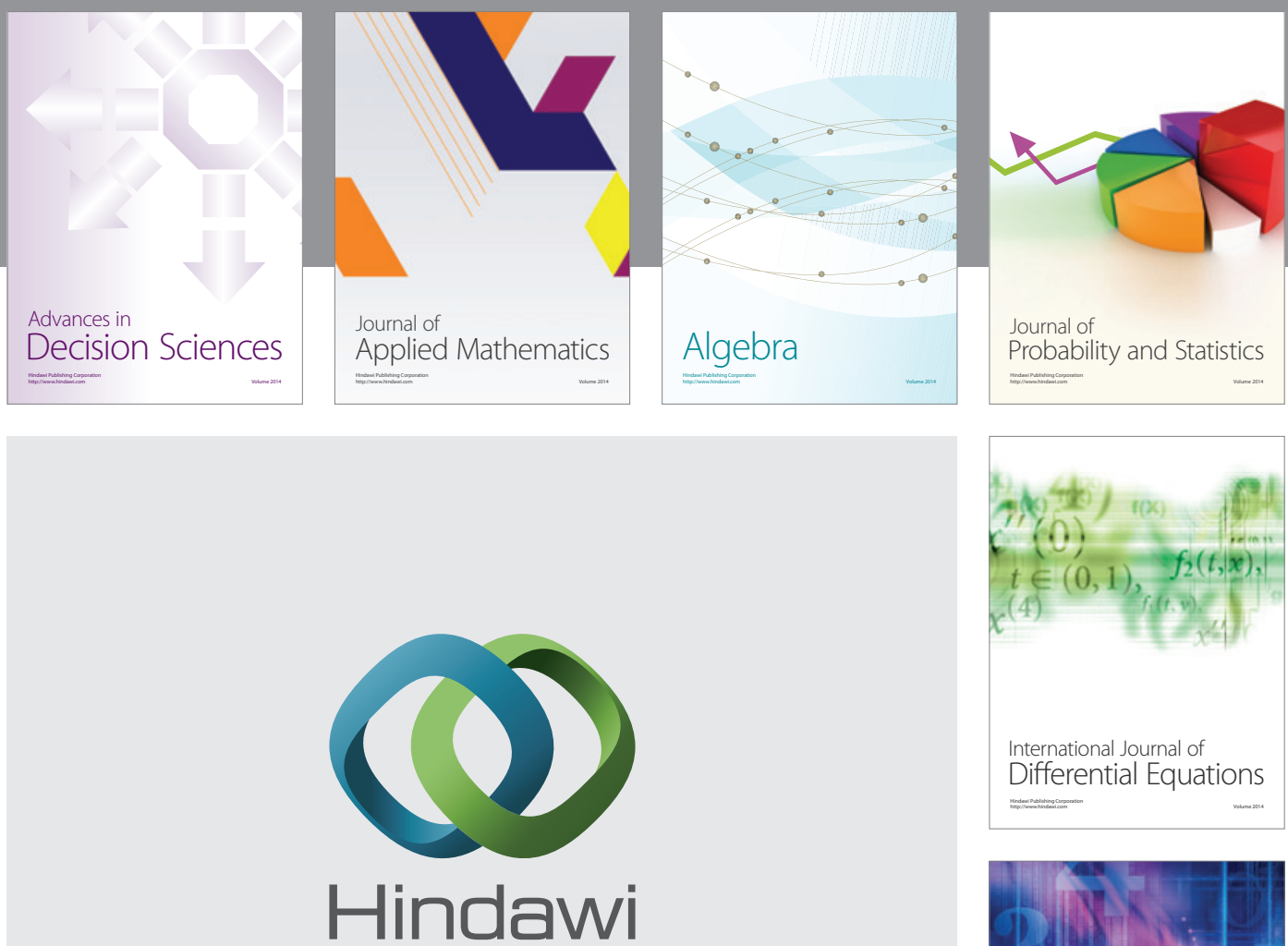

Submit your manuscripts at http://www.hindawi.com
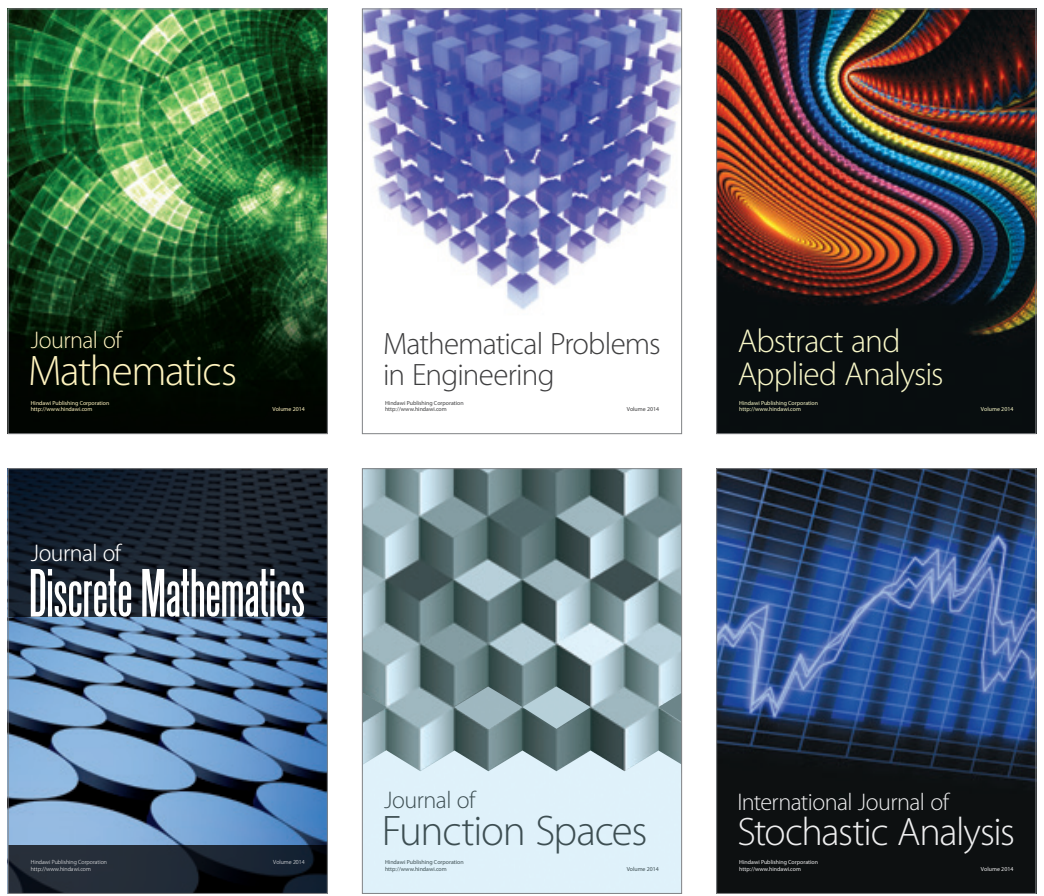

Journal of

Function Spaces

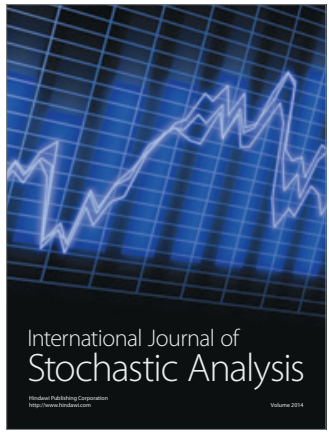

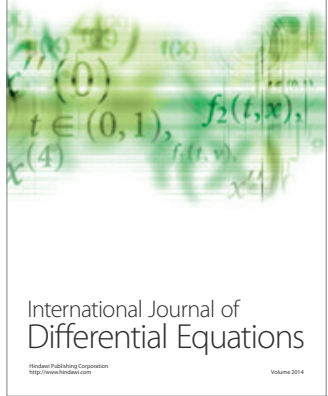
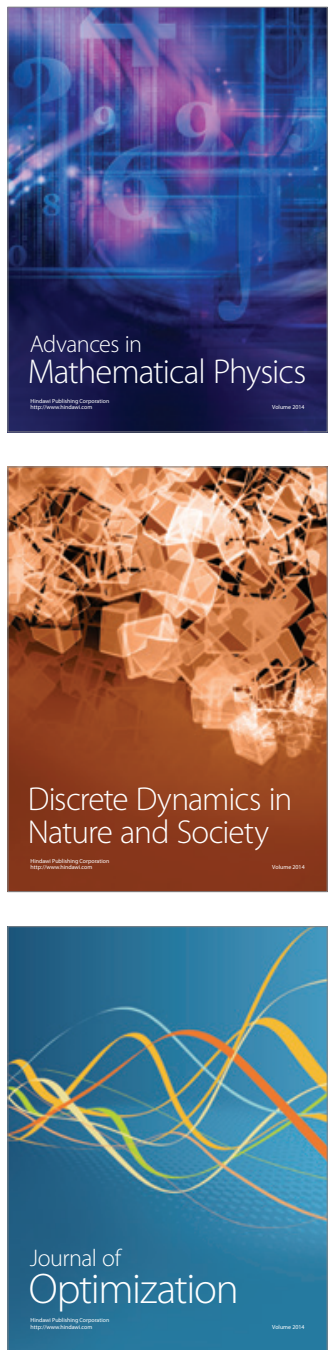